\title{
The relaunch of IJPH's knowledge synthesis, translation, and exchange section
}

\author{
Erica Di Ruggiero'
}

Received: 5 March 2018 / Accepted: 7 March 2018/Published online: 13 March 2018

(c) Swiss School of Public Health (SSPH+) 2018

In 2011, Potvin and Abel's Editorial launched a new section on knowledge synthesis, translation, and exchange (KSTE) for IJPH. 7 years later, the Journal is seeking to further increase the number and diversity of submissions with relevance to public health. At the core of this effort is continued openness to publish manuscripts, commentaries, and editorials that span the breadth of KSTE and pertain to public health. Scholarship that applies different methods of synthesis and evaluates diverse knowledge translation and exchange strategies for improving evidence-informed decision-making in public health has exploded in recent decades (Di Ruggiero et al. 2017). Research efforts in this realm are inherently complex, as they generate, synthesize, and translate public health evidence regarding the determinants of health and health equity alongside interventions (e.g. policies, programs, and laws) that aim to influence the distribution of risk at a population level (Hawe and Potvin 2009). As a result, a plurality of concepts, theories, models, frameworks, and methods is being used-and, in some instances, raising conflicting perspectives about what constitutes "good evidence" in public health. Their application also raises fundamental questions about who and what, and under what contextual circumstances knowledge is generated, disseminated, and used or not, and with what kind of measurable impacts. For instance, whose decisions are actually being influenced and how? At what level (e.g., individual, organizational/institutional, network, and/or system levels), and in which sectors (within and outside of health)? What is the relative contribution of the historical, social, cultural, institutional, and/or political context in public health decision-making? While generally poorly

This editorial is part of the section "Knowledge Synthesis, Translation and Exchange".

Erica Di Ruggiero

e.diruggiero@utoronto.ca

1 Dalla Lana School of Public Health, University of Toronto, Toronto M5T3M7, Canada described, attention to context, how it is characterized as a phenomenon (through the application of appropriate theories, for example), how it is treated in the synthesis of knowledge, and elucidated through high-quality empirical studies to shed light on the contextual influences of knowledge use, is another topic requiring further study (Shoveller et al. 2016). There are also questions related to the ethics of KSTE, drawing much needed attention to the role of values, assumptions, culture, power, and voice in decision-making. Finally, research addressing equity considerations, including social gradients in intervention effects, is crucial to monitoring global, national, and subnational public health interventions (Welch et al. 2013). With this broad set of questions and issues as a backdrop, the IJPH is pleased to relaunch its KSTE section:

Systematic reviews, meta-syntheses and meta-analyses, narrative reviews, realist reviews, scoping reviews, and practice guidelines are all examples of knowledge syntheses using qualitative and/or quantitative methods that need to be reproducible and transparent. The Journal invites submissions that rigorously apply any of these review methods with relevance to public health and related interventions. Reviews can follow methods set out by the Cochrane Collaboration, including its Public Health Group, the Campbell Collaboration) or other synthesis approaches such as realist reviews (Pawson et al. 2005) and scoping reviews (Arksey and O'Malley 2005). Each can present different challenges related to the state, heterogeneity, and complexity of the evidence base being synthesized. The evaluation of knowledge translation and exchange (KTE) strategies, tools, governance mechanisms, and other approaches that can facilitate or hinder the dissemination, utilization, or integration of knowledge into decisionmaking in health and other sectors (e.g.. environment, education) is also an important area of interrogation. Submissions that assess these and other KTE strategies with relevance to public health are also welcomed.

As part of the KTSE section's relaunch, I have invited experts to reflect on substantive issues in the field. Their 
editorials will be published in the coming months, and I hope that they will fuel further dialogue and debate, and encourage a diversity of submissions from different parts of the globe. It is also my hope that this revitalized section will lead to an increase in high-quality conceptual and empirical submissions about the synthesis, translation, and exchange of public health evidence, and help contribute to evidence-informed and equity-oriented public health policy and practice.

\section{Erica Di Ruggiero, MHSc PhD RD.}

Associate Editor, International Journal of Public Health.

\section{References}

Arksey H, O’Malley L (2005) Scoping studies: towards a methodological framework. Int J Soc Res Methodol 8(1):19-32
Di Ruggiero E, Viehbeck S, Greyson D (2017) Knowledge utilization and exchange in public health. Oxford Bibliographies in Public Health

Hawe P, Potvin L (2009) What is population health intervention research? Can J Public Health 100(1):8-14

Pawson R, Greenhalgh T, Harvey G, Walshe K (2005) Realist review - a new method of systematic review designed for complex policy interventions. J Health Serv Res Policy 10(1):21-23

Potvin L, Abel T (2011) A new section for IJPH: knowledge synthesis, translation and exchange. Int J Public Health 56(1). https://doi.org/10.1007/s00038-010-0218-4

Shoveller J, Viehbeck S, Di Ruggiero E, Greyson D, Thomson K, Knight R (2016) A critical examination of representations of context within research on population health interventions. Crit Public Health 26(5):487-500

Welch VA, Petticrew M, O'Neill J, Waters E, Armstrong R, Bhutta ZA, Francis D, Perez Koehlmoos T, Kristjansson E, Pantoja T, Tugwell P (2013) Health equity: evidence synthesis and knowledge translation methods. Syst Rev 2:43 